\title{
Liver Biochemical Abnormalities in Turner Syndrome: a Comprehensive Characterisation of an Adult Population
}

Short title: Liver enzymes in Turner syndrome

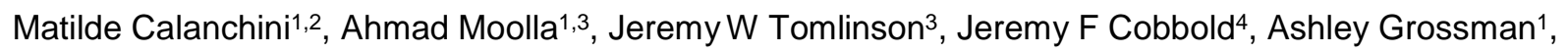
Andrea Fabbri², Helen E Turner ${ }^{1}$

${ }^{1}$ Oxford Centre for Diabetes, Endocrinology \& Metabolism, University of Oxford, Churchill Hospital, Oxford, United Kingdom; ${ }^{2}$ Department of Systems Medicine, Endocrinology \& Metabolism Unit, University of Rome Tor Vergata, Rome, Italy; ${ }^{3}$ Oxford Centre for Diabetes, Endocrinology \& Metabolism, National Institute for Health Research, Oxford Biomedical Research Centre, University of Oxford, Churchill Hospital, Oxford, United Kingdom; ${ }^{4}$ Department of Gastroenterology and Hepatology, Oxford University Hospitals NHS Trust, Oxford, United Kingdom

\section{Declaration of interest}

The authors report no conflicts of interest in this work.

\section{Funding}

This research did not receive any specific grant from any funding agency in the public, commercial or not-forprofit sector.

\section{Acknowledgments}

The research was supported by the National Institute for Health Research (NIHR) Oxford Biomedical Research Centre (BRC).

Disclaimer: The views expressed are those of the authors and not necessarily those of the NHS, the NIHR or the Department of Health. 


\section{Summary}

Objective: Abnormal liver function tests (LFTs) are frequent in Turner syndrome (TS). The causes and clinical significance are unclear. Aims: to investigate the prevalence of elevated LFTs in adult TS; secondly, to analyse the associations between elevated LFTs, TS-karyotypes, and TS-related conditions; and thirdly, to evaluate liver stiffness and histological assessment.

Methods: 125 TS women were retrospectively studied. Karyotypes, clinical and biochemical details and aortic measurements were recorded. Fibroscan and liver biopsy results were noted.

Results: Elevated LFTs were found in $49.6 \%$ : GGT in $88.7 \%$, ALK in $45.2 \%$, ALT in $40.3 \%$ and AST in $29 \%$. A FIB-4 index $>1.3$ was found in $11.8 \%$. Women with isochromosome of the $X$ long arm, iso[X](q), had a higher prevalence of elevated LFTs. A lower prevalence of abnormal GGT was found in patients with $45, X / 46, X X, 45, X / 47, X X X$ or $45, X / 46, X X / 47, X X X$.

Subjects with raised GGT were older, shorter and more likely to have higher triglyceride levels. There was no association with HRT duration after adjusting for age. Among patients with elevated aminotransferases no differences were noted, except for higher HDL-cholesterol levels. The sinuses and ascending aorta diameter were greater in the elevated LFTs-group.

Fibroscan was suggestive of significant liver fibrosis in $38.1 \%$. Among 11 biopsies, liver architectural changes were reported in $45.4 \%$, including two with cirrhosis.

Conclusions: Elevated LFTs in TS are common and important to detect given the possible progression towards severe liver disease. An association between raised LFTs and karyotype iso[X]q was demonstrated. We have also shown a new association between abnormal LFTs and aortic dilatation.

Keywords: Turner syndrome, liver enzymes, karyotype, NAFLD, aortic diameter, liver biopsy

\section{Introduction}

Turner syndrome (TS) is the most common sex-chromosome disorder in females, affecting 1:2500 live-born. It is due to a range of karyotypes, from complete or partial loss, to complex rearrangements affecting the $X$ chromosome. While short stature and gonadal dysgenesis are the commonest characteristics, there is a broad clinical spectrum including congenital heart cardiovascular disease and predisposition to autoimmune disease. ${ }^{1}$

Abnormal liver function tests (LFTs) are frequent in TS. The reported prevalence of elevated LFTs ranges 
from $20-80 \%$, and rises with age..$^{2,3-5}$ Although many have considered this to be a benign biochemical abnormality, some studies have suggested a more cautious approach. ${ }^{6-9}$ Nodular regenerative hyperplasia and cirrhosis have been described in TS, along with the more common condition of nonalcoholic fatty liver disease (NAFLD). ${ }^{6-9} \mathrm{~A}$ ten year follow-up study in TS women reported a six-fold increased incidence of cirrhosis, ${ }^{10}$ while a large study investigating cause of death found a three-fold increase in liver disease mortality in TS compared to the general population. ${ }^{11}$ Furthermore, biliary lesions, including bile duct paucity, biliary atresia, sclerosing cholangitis and primary biliary cirrhosis, have been described. ${ }^{7,8}$ Several pathophysiological mechanisms to explain the abnormal LFTs in TS patients have been proposed. While oestrogen hormone replacement therapy (HRT) is not associated with elevated liver enzymes per se, 5,12 excess weight is one of the common causes thought to contribute to this finding. ${ }^{4,5}$ Previous studies investigating elevated LFTs and autoimmunity have yielded controversial results. ${ }^{4,5,13,14}$ Recently, an association between higher alanine aminotransferase (ALT) levels and ring $X$ karyotype was found in a large TS population, ${ }^{15}$ whereas prior studies had shown no association with TS-karyotypes. Finally, cohort studies have suggested that the architectural liver changes described in TS may be the consequence of a primary vascular derangement. ${ }^{7}$ This would be compatible with other common vascular changes seen in TS, such as aortic dilatation and increased intima media vessel wall thickness. ${ }^{16,17}$

Thus, the pathogenesis and the clinical significance of LFT elevation are still unclear. The aim of this study was, firstly, to investigate the prevalence of liver biochemical abnormalities in a large TS population and, in order to improve the detection of liver disease, we used the more stringent lower ALT cut-off for women ${ }^{18,19}$ and the Fibrosis-4 (FIB-4) score..$^{20}$ Secondly, in order to elucidate the possible pathophysiological mechanisms underlying the liver abnormalities, we analysed the associations between elevated LFTs and different TS-karyotypes, and between abnormal LFTs and other conditions more commonly seen in TS. Thirdly, we investigated the extent to which women with elevated LFTs have any structural hepatic changes, evaluating liver stiffness using transient elastography and histological assessment, in order to establish the actual clinical significance of LFTs abnormalities in TS.

\section{Subjects and Methods}

\section{A. Patients and Liver Biochemical Evaluation}

We conducted a retrospective service evaluation of 125 women attending our adult TS clinic. All data were collected as part of routine care for patients with TS. Women with measurements of aminotransferases (ALT 
and aspartate transferase, AST), gamma-glutamyl transferase (GGT) and alkaline phosphatase (ALP), on at least two occasions, were included. Patients with a history of excessive alcohol consumption (>14 alcoholic units/week) or on medications that significantly raise LFTs, were excluded.

Liver enzymes were defined as abnormal when higher than the upper limit of normal (ULN) (ALT >45 IU/L, AST $>42 \mathrm{IU} / \mathrm{L}, \mathrm{GGT}>40 \mathrm{IU} / \mathrm{L}$ and $\mathrm{ALP}>130 \mathrm{IU} / \mathrm{L}$ ) for more than 6 months. The more stringent analysis used an ALT cut-off of $19 \mathrm{IU} / \mathrm{L} .^{18}$

The AST/ALT ratio and the aspartate-to-platelet ratio index (APRI) were calculated, with an APRI score $>0.8$ considered as cut-off for predicting significant fibrosis. ${ }^{21}$ The presence of advanced hepatic fibrosis was assessed using the FIB-4 index, which is determined using transaminase levels, platelet counts and age. Scores of FIB-4<1.3 were classified as suggestive of low risk of advanced fibrosis and scores $>2.67$ were classified as high risk. ${ }^{20}$

Patients with unexplained elevation of aminotransferases underwent a liver screen to exclude common causes of liver disease to include: serum immunoglobulin levels, antinuclear antibodies and liver autoantibodies, hepatitis B surface antigen, anti-hepatitis $C$ antibody, ferritin and transferrin saturation, ceruloplasmin and alpha-1 antitrypsin.

\section{B. Genetic, Clinical and Biochemical Evaluation}

Karyotype analysis was performed using lymphocyte cultures in at least 30 cells. TS-karyotypes were classified into eight groups: 1) monosomy $X, 45, X ; 2)$ mosaicism with a second or third cell line with more than one $X, 45, X / 46, X X, 45, X / 47, X X X$ and 45,X/46,XX/47,XXX; 3) deletion of the $X$ short arm, del[X](p); 4) deletion of the $X$ long arm, $\operatorname{del}[X](q) ; 5)$ isochromosome of the $X$ long arm, iso $[X](q)$; 6) ring $X, r[X] ; 7)$ presence of whole or partial Y chromosome and 8) other TS-karyotypes. The groups from 3 to 8 included mosaic and non-mosaic karyotypes.

Body height, weight and body mass index (BMI) were collected. Body surface area was calculated using the Du Bois formulas. ${ }^{22}$ Diabetes, dyslipidaemia and hypertension status were defined by past-medical history or standard criteria. ${ }^{23}$ Details regarding cardiac surgery, growth hormone (GH) therapy, HRT and thyroxine treatment were recorded. Full blood count, lipids, HbA1c, albumin, bilirubin, 25-hydroxyvitamin D (25OHD), thyroid-stimulating hormone (TSH) and free thyroxine (FT4) were measured, as per TS routine annual assessment. ${ }^{23}$ Thyroid peroxidase antibodies (TPOAb) and screening for celiac disease were performed at diagnosis and every 5 years or in the presence of suggestive symptoms. ${ }^{23}$ 


\section{Cardiovascular Assessment}

Cardiac assessment, performed by TS-expert cardiologists, including transthoracic echocardiography (repeated at least every three years) and magnetic resonance imaging (MRI) (repeated every 5-10 years and before planned pregnancy), was available in 120 women. Information on cardiac malformations, dimension of the ascending aorta and aortic sinuses, were collected. As short stature is common in TS and body size is a major determinant of normal aortic dimensions, aortic size was adjusted for body surface area and reported as the aortic size index (ASI). An ASI $>2 \mathrm{~cm} / \mathrm{m}^{2}$ was used to define the presence of aortic dilatation. ${ }^{23,24}$

\section{Liver Imaging and Histological Evaluation}

Hepatic ultrasound and liver stiffness measurement (LSM) using transient elastography (Fibroscan; Echosens, Paris, France) were collected. Fibroscan is used for the assessment of liver fibrosis. It measures the velocity of a low-frequency elastic shear wave propagating through the liver. This velocity is directly related to tissue stiffness. LSM $>8 \mathrm{kPa}$ was used as a cut-off for significant liver fibrosis and $>13 \mathrm{kPa}$ to suggest cirrhosis. ${ }^{25}$ LSM was considered reliable when it included ten valid measurements with a success rate of at least $60 \%$ and interquartile range/median value $<0.30$. In presence of a $\mathrm{BMI}>28 \mathrm{~kg} / \mathrm{m}^{2}$ the XL probe was used.

Liver biopsy was performed in women with unexplained elevation of aminotransferases that persisted for $>1$ year in presence of clinical and radiological findings associated with an increased risk for steatohepatitis and/or advanced fibrosis. The grade of steatosis, the NAFLD activity score (NAS) ${ }^{26}$ and the Ishak fibrosis stage ${ }^{27}$ were collected. Nodular regenerative hyperplasia, focal nodular hyperplasia and cirrhosis were collectively regarded as marked architectural changes.

The liver findings were reviewed in a multidisciplinary meeting by hepatologists, radiologists, pathologists and endocrinologists with a special interest in TS and/or liver disease.

\section{E. Statistical Analysis}

Data are presented as mean, standard deviations (SD) and median values. Patients with abnormal LFTs were subdivided into two groups: women with elevated GGT and women with elevated aminotransferases and/or FIB-4 score. Student's $t$-test, Fisher's exact test and chi-square test were used to calculate 
differences between groups. Simple correlations were assessed using Pearson's correlation coefficient. $P$ value $<0.05$ was considered statistically significant. All statistical analyses were performed with SPSS statistical software version 11.0 (SPSS Inc., Chicago, IL, USA).

\section{Results}

\section{A. Prevalence of Liver Abnormalities}

A total of 125 TS women, with a median age of 35 years, were studied; their clinical characteristics are summarised in Table 1. Elevated LFTs were found in $49.6 \%$ of patients. Among those women, abnormal GGT was found in $88.7 \%$, elevated ALK in $45.2 \%$, elevated ALT in $40.3 \%$ and elevated AST in $29 \%$. Using the ALT cut-off of $19 \mathrm{UI} / \mathrm{L}, 69.6 \%$ of patients had an elevated ALT.

Raised AST, ALT and/or a FIB-4 score $>1.3$ were found in $26.4 \%$ of women, and in this group the median (range) LFT results were ALT 63 (11-286) UI/L, AST 43 (18-157) UI/L, FIB-4 0.96 (0.43-2.7) and GGT 153 (10-603) UI/L, with a median AST/ALT ratio of 0.73 (0.36-1.93) and APRI 0.39 (0.11-1.61). A FIB-4 index $>1.3$ (range 1.3-2.67) was found in $11.8 \%$ of women; half of them had normal aminotransferase levels. An APRI $>0.8$ was found in $2.4 \%$.

Increased liver enzymes were first detected at a median age of 32 (range 15-71) years and were persistently elevated during a median follow-up of 3 (range 1-31) years.

\section{B. Association Study}

\section{Elevated liver biochemistry and TS-karyotypes}

Among patients with elevated GGT, the prevalence of TS-karyotypes were: $81.8 \%$ for iso[X]q; $71.4 \%$ in the group with presence of $\mathrm{Y} ; 54.3 \%$ for $45, \mathrm{X} ; 50 \%$ for del[X]p and del[X]q; $36.4 \%$ for $r[\mathrm{X}]$ and $12.5 \%$ for $45, \mathrm{X} / 46, \mathrm{XX}, 45, \mathrm{X} / 47, \mathrm{XXX}$ or $45, \mathrm{X} / 46, \mathrm{XX} / 47, \mathrm{XXX}$. Women with iso[X]q were significantly more common within the elevated GGT group than the normal: $16.7 \%(9 / 54)$ vs $3.8 \%(2 / 53), P=0.028$ (Figure 1A). Also, patients with iso[X]q showed a higher level of GGT compared to the subjects with other TS-karyotypes (226.1 \pm 182.5 vs $76.6 \pm 98.6 \mathrm{UI} / \mathrm{L}, P=0.022$ ). Conversely, a lower prevalence of raised GGT was found in women with $45, \mathrm{X} / 46, \mathrm{XX}, 45, \mathrm{X} / 47, \mathrm{XXX}$ or $45, \mathrm{X} / 46, \mathrm{XX} / 47, \mathrm{XXX}$ mosaicism ( $3.7 \%$ vs $26.4 \%, P<0.001)$, with a lower level of GGT compared to other karyotypes $(27.1 \pm 35$ vs $103.3 \pm 123.8 \mathrm{UI} / \mathrm{L}, P<0.001)$. 
Among subjects with elevated aminotransferases/FIB-4, the prevalence of TS-karyotypes were: $58.3 \%$ for iso[X]q; $50 \%$ for del[X]p; $36.4 \%$ for $r[X] ; 29.4 \%$ for $45, X ; 11.1 \%$ for $45, X / 46, X X, 45, X / 47, X X X$ or $45, X / 46, X X / 47, X X X$ and $0 \%$ in the group of women with del[X]q and presence of $Y$.

Women with iso[X]q were a higher proportion of the elevated aminotransferases/FIB-4 group compared with the normal group: $22.6 \%(7 / 31)$ vs $5.7 \%(5 / 87), P=0.014$ (Figure 1B).

In view of the higher prevalence of iso[X]q in subjects with raised LFTs, this karyotype group was further studied. All clinical and biochemical characteristics of women with iso[X]q were similar to the group of patients with other TS-karyotypes; in particular no differences were found for type 2 diabetes and autoimmune disease.

\section{Elevated liver biochemistry and TS-related conditions}

Liver screen tests to exclude common causes of liver disease were normal in all cases. The characteristics of patients with an elevated GGT are summarised in Table 2. Women with raised GGT were older (40.4 \pm 14.6 vs $33.1 \pm 11.8$ years, $P=0.004)$, shorter $(147.8 \pm 6.8$ vs $151.7 \pm 7.3 \mathrm{~cm}, P=0.004)$ and more likely to have higher triglyceride levels ( $1.4 \pm 0.8$ vs $1.1 \pm 0.6 \mathrm{mmol} / \mathrm{L}, P=0.044)$. HRT duration was longer in the subjects with elevated GGT; however, after adjusting for age, HRT duration was not significantly different between the two groups. No differences were noted for weight, BMI, $\mathrm{HbA}_{1} \mathrm{C}, \mathrm{TSH}, \mathrm{FT} 4$ or $25 \mathrm{OHD}$. Similarly, there were no differences in the prevalence of diabetes, hypertension, dyslipidaemia, autoimmune disease, previous GH therapy, cardiac or renal congenital abnormalities between the group of women with normal or raised GGT. On analysis of the cardiac data, the ascending aorta diameter was higher in subjects with an elevated GGT ( $2.94 \pm 0.55$ vs $2.68 \pm 0.38 \mathrm{~cm}, P=0.007$, and ASI $1.9 \pm 0.41$ vs $1.72 \pm 0.3 \mathrm{~cm} / \mathrm{m}^{2}, P=0.011$ ) and likewise, for the absolute diameter of the aortic sinuses ( $2.95 \pm 0.38$ vs $2.8 \pm 0.34 \mathrm{~cm}, P=0.037)$.

The characteristics of patients with elevated aminotransferases/FIB-4 are reported in Table 3. No differences were noted, except for lower HDL-cholesterol levels in subjects with normal aminotransferases $(1.7 \pm 0.5 \mathrm{vs}$ $1.9 \pm 0.5 \mathrm{mmol} / \mathrm{L}, P=0.043$ ). Also, no associations were found considering the group of women with $\mathrm{ALT}>19$ $\mathrm{UI} / \mathrm{L}$, except for lower height (148.4 \pm 7.9 vs $152.9 \pm 7.2 \mathrm{~cm}, P=0.003)$. Both the ASI of the ascending aorta and sinuses were higher in subjects with raised aminotransferases $\left(2.02 \pm 0.32 \mathrm{vs} 1.83 \pm 0.39 \mathrm{~cm} / \mathrm{m}^{2}\right.$, $P=0.032$, and for aortic sinuses $2.02 \pm 0.35$ vs $\left.1.82 \pm 0.32 \mathrm{~cm} / \mathrm{m}^{2}, P=0.025\right)$.

\section{Liver Imaging and Biopsy Study}


Liver ultrasonography was available in 29 women with abnormal LFTs, showing increased echogenicity with fatty infiltration in $32 \%$. A Fibroscan was performed in $63.6 \%$ (21/33) of patients with raised aminotransferases: a LSM suggestive of fibrosis was found in $38.1 \%$; of these two women had LSM $>13$ $\mathrm{kPa}$.

Fourteen patients were referred for a liver biopsy and three refused the biopsy. Median age at first biopsy was 38 (20-56) years. Liver architectural changes were reported in 5 patients (Table 4). One woman was diagnosed with cirrhosis and the biopsy showed capillarisation of the sinusoids, sinusoidal dilatation and paucity of the bile ducts. Splenomegaly was present, but no evidence of varices were found. Her LSM was $30.1 \mathrm{kPa}$. A second woman, with an elevated FIB-4 index, was diagnosed with cirrhosis and severe steatosis, although normal LSM. Two patients presented with nodular regenerative hyperplasia and mild fibrosis. Lastly, one woman had focal nodular regenerative hyperplasia.

Among subjects with no architectural changes, steatosis and fibrosis were common (Table 4). One woman had histopathological evidence of biliary injury; at magnetic resonance cholangiopancreatography, mild intrahepatic and moderate extrahepatic common bile duct dilatation was found with no focal mass or stones in the duct at the endoscopic cholangiopancreatography.

Finally, one patient (patient 8 in Table 4 and 5) with LSM value suggestive of cirrhosis presented with severe steatosis and only mild fibrosis. Her BMI was $44 \mathrm{~kg} / \mathrm{m}^{2}$ and, although the XL probe was used, LSM was regarded as a false positive result.

The clinical features of the patients undergoing liver biopsy are summarised in Table 5. Among women with liver architectural changes and biliary involvement, aortic dilatation was present in four, and four had cardiac congenital abnormalities.

\section{Discussion}

\section{A. Prevalence of Liver Abnormalities}

In our large population of adult TS women, followed in a single centre, about $50 \%$ had abnormal liver biochemistry, which was most commonly an elevated GGT. Using a more sensitive ALT cut-off, ${ }^{18}$ we found that $70 \%$ had an elevated ALT. Recent evidence suggests that the upper limit threshold of ALT level should be lowered, since subjects who have raised ALT values which are still within the standard ULN have an increased risk of mortality from liver disease. ${ }^{19}$ It has been also proposed that gender-specific thresholds should be applied, since women have lower normal ALT levels than men. ${ }^{18}$ Thus, we suggest consideration 
of a lower threshold for ALT in TS patients.

One of the strengths of this study is the analysis of the complete panel of LFTs during a median follow-up of three years. Including both aminotransferases, we noticed that a common finding was a moderate elevation of transaminases, with slightly higher levels of ALT than AST, as usually seen in patients with chronic liver disease without advanced fibrosis. In addition, median APRI and FIB-4 values in subjects with raised LFTs were suggestive of a low risk of advanced fibrosis. However, $12 \%$ of women had FIB-4 values $>1.3$, even in the presence of normal aminotransferases. Therefore, we support the use of FIB-4 in TS patients as a simple and non-invasive biochemical test for excluding advanced liver fibrosis.

\section{B. Association Study}

\section{Elevated liver biochemistry and TS-karyotypes}

We have demonstrated an association between TS-karyotype and abnormal LFTs, whereas the majority of previous studies have failed to demonstrate any significant relationship. One explanation might be that the previous studies pooled together different karyotypes, but we precisely subdivided TS-karyotypes into eight groups. We found a lower prevalence of elevated GGT in women with mosaicism 45,X/46,XX, 45,X/47,XXX or $45, X / 46, X X / 47, X X X$, and an increased prevalence of abnormal LFTs in subjects with the iso[X]q. Importantly, the iso[X]q is the most common structural abnormality in TS. It results from faulty meiotic recombination events involving pericentromeric repetitive sequences, leading to deletion of most of the short arm (Xp) and duplication of the long arm (Xq). As such, in an attempt to identify the chromosomal locus for the liver biochemical abnormalities in TS, we speculate that subjects with deletion of the short arm of the $X$ are more prone to LFT elevation. Indeed, we found that women with iso[X]q, deletion of the short arm and 45,X karyotypes had a higher prevalence of LFT abnormalities compared to women with deletion of the long arm of the $\mathrm{X}$ and $45, \mathrm{X} / 46, \mathrm{XX}, 45, \mathrm{X} / 47, \mathrm{XXX}$ or $45, \mathrm{X} / 46, \mathrm{XX} / 47, \mathrm{XXX}$ mosaicism. The lack of a significant prevalence of subjects with del[X]p within the group with elevated LFTs might be explained by the small sample size of this karyotype group in our population. Indeed, both $\mathrm{Xq}$ and $\mathrm{Xp}$ deletion karyotypes are less frequent. Thus, further observations are needed to confirm the hypothesis that Xq deletion women are less at risk for LFT abnormalities.

Since an association between the iso[X]q and TPOAb positivity was previously reported, ${ }^{28}$ even if not always confirmed, ${ }^{29}$ we addressed this point in our population, but no association between the iso[X]q and TPOAb and autoimmune disease overall was found. In addition, we found that subjects with iso[X]q had similar 
features compared with other TS-karyotypes.

Thus, the genetic explanation for the higher prevalence of LFT abnormalities in TS is currently unclear, but our preliminary data suggest that further investigation of the iso[X]q might help elucidate a mechanism.

\section{Elevated liver biochemistry and TS-related conditions}

In order to better understand the liver involvement in TS, we analysed LFT abnormalities distinguishing patients with raised GGT from the group with elevated aminotransferases. Indeed, abnormal aminotransferases are considered the most useful measures of liver cell injury. We found that advanced age, lower stature and higher triglyceride levels were more frequent in women with elevated GGT, whereas higher HDL-cholesterol levels were more common in patients with elevated aminotransferases. Notably, the shorter stature of women with abnormal GGT was not associated with a delayed start of GH treatment due to a later diagnosis of TS. Interestingly, a recent study reported an unexpectedly high frequency of chromosome $\mathrm{X}$ abnormalities in women, not previously diagnosed with TS, with short stature and elevated LFTs. ${ }^{30}$ Although several studies quote BMI as the most important contributing factor to the development of liver abnormalities in TS, we did not find any difference regarding weight or BMI between women with normal versus elevated LFTs.

Previous studies reported an association between LFT abnormalities and autoimmune hepatitis, thyroid antibodies and coeliac disease..$^{13,14}$ However, in our TS population, liver specific autoimmunity, thyroid autoimmunity and coeliac disease were not overrepresented in women with abnormal LFTs. We also found that low $25 \mathrm{OHD}$ levels, which have been associated with autoimmune diseases ${ }^{31}$ and NAFLD ${ }^{32}$ in the general population, were not more common in TS women with liver abnormalities.

Importantly in this study, a longer duration of HRT was not associated with raised LFTs after adjusting for age. Thus, in accordance with previous studies, we recommend that HRT be continued in women with elevated LFTs. The majority of women were on transdermal oestrogen or received transdermal oestrogen replacement therapy before reaching the menopausal age, but many of those had been previously treated with oral oestrogen. For these reasons, a study of the difference between transdermal versus oral oestrogen on LFTs was not performed.

For the first time, we report an association between abnormal LFTs and increased aortic diameter. It is well known that TS is associated with a range of cardiovascular comorbidities, including valvular heart disease, coarctation of the aorta and aortic dilatation. Some studies suggest that the aorta may not be the only vessel affected in TS, suggesting a general vasculopathy. ${ }^{16,17,33}$ Accordingly, in Roulot's study, which is the largest 
study on liver biopsy in TS, it was proposed that some hepatic changes might be part of a general vascular disorder. ${ }^{7,8}$ Indeed, nodular regenerative hyperplasia is thought to represent a form of adaptation to microcirculatory disturbances causing heterogeneous distribution of intrahepatic blood flow, ${ }^{34}$ and cirrhosis in TS patients may correspond to the final stage of this vascular disorder. Our aortic measurements in women with abnormal LFTs support this hypothesis, but larger studies are needed to corroborate these findings.

\section{Liver Imaging and Biopsy Study}

To further establish the significance of abnormal LFTs and in particular to assess fibrosis, we measured liver stiffness using transient elastography. We found that raised LFTs were more commonly detected in TS subjects with higher LSM. The only previous study with Fibroscan in TS involved 25 young patients and showed that LSM values were correlated with liver enzyme levels. ${ }^{35}$ Given that Fibroscan is a repeatable and non-invasive technique to evaluate liver stiffness, and since liver involvement in TS might be present at a relative young age, there is a need to conduct larger studies in order to define the utility of Fibroscan among TS patients.

This study is the second largest study on liver biopsy in TS. Consistent with Roulot's study (27 biopsies) ${ }^{7}$, we reported a variety of histological appearances. Approximately $50 \%$ of women in whom a liver biopsy was performed were found to have significant abnormalities. Interestingly, in Roulot's study the most serious pathology occurred in women who had congenital cardiac abnormalities, but aortic diameters were not reported. ${ }^{7}$ In our cohort about half of the women with major liver architectural changes had cardiac abnormalities and aortic dilatation.

Taking liver biopsy as the reference standard, about half of the subjects who had a liver biopsy would have been inappropriately referred or not referred to a liver biopsy when FIB-4, APRI and Fibroscan were considered individually. However, there were insufficient numbers of liver biopsies thus far to determine whether a combination of FIB-4/APRI/Fibroscan may be reliable non-invasive investigations to delineate those women at risk of cirrhosis.

In conclusion, elevated LFTs are common in TS and important to detect given the high prevalence and clinical relevance of possible progression towards advanced fibrosis. For the first time, an association between raised LFTs and iso[X]q karyotype was found. We have also reported an association between abnormal LFTs and aortic dilatation, suggesting that liver involvement may be related to a primary vascular process. From a clinical point of view, these findings highlight the importance of multidiscliplinary 
management of liver abnormalities in TS. An annual monitoring of LFTs is recommended and a reduced threshold for ALT and the use of FIB-4 score is suggested in order to detect TS women with hepatic abnormalities. We suggest that surveillance using non-invasive imaging techniques, such as liver ultrasonography and Fibroscan, should be adopted in TS patients with abnormal biochemical results and the indication for liver biopsy evaluated according to the clinical context. Women with karyotypes involving a deletion of the short arm of the $\mathrm{X}$ and with aortic dilatation are potentially more at risk for developing liver test abnormalities and further studies are needed to confirm these results.

\section{References}

1. Gravholt CH. Epidemiological, endocrine and metabolic features in Turner syndrome. Eur J Endocrinol. $2004 ; 151: 657-687$.

2. Sylven L, Hagenfeldt K, Brondum-Nielsen K \& von-Schoultz B. Middle-aged women with Turner's syndrome. Medical status, hormonal treatment and social life. Acta Endocrinol. 1991; 125 359-365.

3. Albareda MM, Gallego A, Enriquez J, Rodriguez JL \& Webb SM. Biochemical liver abnormalities in Turner's syndrome. Eur J Gastroenterol Hepatol. 1999; 11: 1037-1039.

4. El-Mansoury M, Berntorp K, Bryman I, et al. Elevated liver enzymes in Turner syndrome during a 5-year follow-up study. Clin Endocrinol (Oxf). 2008; 68: 485-490.

5. Koulouri O, Ostberg J \& Conway GS. Liver dysfunction in Turner's syndrome: prevalence, natural history and effect of exogenous oestrogen. Clin Endocrinol (Oxf). 2008; 69: 306-310.

6. Idilman R, De Maria N, Colantoni A, Kugelmas M \& Van Thiel DH. Cirrhosis in Turner's syndrome: case report and literature review. Eur J Gastroenterol Hepatol. 2000; 12: 707-709.

7. Roulot $D$, Degott $C$, Chazouillères $O$, et al. Vascular involvement of the liver in Turner's syndrome. Hepatology. 2004; 39: 239-247.

8. Roulot D. Liver involvement in Turner syndrome. Liver Int. 2013; 33: 24-30.

9. Kawabata S, Sakamoto S, Honda M, et al. Liver transplantation for a patient with Turner syndrome presenting severe portal hypertension: a case report and literature review. Surg Case Rep. 2016; 2: 68.

10. Gravholt CH, Juul S, Naeraa RW \& Hansen J. Morbidity in Turner syndrome. J Clin Epidemiol. 1998; 51 : 147-158. 
11. Schoemaker MJ, Swerdlow AJ, Higgins CD, Wright AF, Jacobs PA; United Kingdom Clinical Cytogenetics Group. Mortality in women with Turner Syndrome in Great Britain: A National Cohort Study. J Clin Endocrinol Metab. 2008; 93: 4735-4742.

12. Elsheikh M, Hodgson HJ, Wass JA \& Conway GS. Hormone replacement therapy may improve hepatic function in women with Turner's syndrome. Clin Endocrinol (Oxf). 2001; 55: 227-231.

13. Larizza D, Locatelli M, Vitali L, et al. Serum liver enzymes in Turner syndrome. Eur J Pediatr. 2000; 159: 143-148.

14. Salerno M, Di Maio S, Gasparini N, Rizzo M, Ferri P \& Vajro P. Liver abnormalities in Turner syndrome. Eur J Pediatr. 1999; 158: 618-623.

15. Cameron-Pimblett A, La Rosa C, King TFJ, Davies MC \& Conway GS. The Turner Syndrome Life Course Project: Karyotype-phenotype analyses across the lifespan. Clin Endocrinol (Oxf). 2017; 87: 532538.

16. Lawson SA, Urbina EM, Gutmark-Little I, Khoury PR, Gao Z \& Backeljauw PF. Vasculopathy in the young Turner syndrome population. J Clin Endocrinol Metab. 2014; 99: 2039-2045.

17. Ostberg JE, Donald AE, Halcox JP, Storry C, McCarthy C \& Conway GS. Vasculopathy in Turner syndrome: arterial dilatation and intimal thickening without endothelial dysfunction. J Clin Endocrinol Metab. 2005; 90: 5161-5166.

18. Prati D, Taioli E, Zanella A, et al. Updated definitions of healthy ranges for serum alanine aminotransferase levels. Ann Intern Med. 2002; 137: 1-10.

19. Kim HC, Nam CM, Jee SH, Han KH, Oh DK \& Suh I. Normal serum aminotransferase concentration and risk of mortality from liver diseases: prospective cohort study. BMJ. 2004; 328-983.

20. McPherson S, Stewart SF, Henderson E, Burt AD, Day CP. Simple non-invasive fibrosis scoring systems can reliably exclude advanced fibrosis in patients with non-alcoholic fatty liver disease. Gut. 2010; 59: 1265-1269.

21. Wai CT, Greenson JK, Fontana RJ, et al. A simple noninvasive index can predict both significant fibrosis and cirrhosis in patients with chronic hepatitis C. Hepatology. 2003; 38: 518-526.

22. Du Bois D \& Du Bois EF. A formula to estimate the approximate surface area if height and weight be known. Nutrition. 1989; 5: 303-311.

23. Gravholt $\mathrm{CH}$, Andersen $\mathrm{NH}$, Conway GS, et al. Clinical practice guidelines for the care of girls and women with Turner syndrome: proceedings from the 2016 Cincinnati International Turner Syndrome Meeting. Eur J Endocrinol. 2017; 177: 1-70. 
24. Matura L, Ho V, Rosing D \& Bondy C. Aortic Dilatation and dissection in Turner syndrome. Circulation. 2007; 116: 1663-1670.

25. Roulot D, Costes JL, Buyck JF, et al. Transient elastography as screening tool for liver fibrosis and cirrhosis in a community-based population aged over 45 years. Gut. 2011; 60: 977-984.

26. Angulo P, Hui JM, Marchesini G, et al. The NAFLD fibrosis score: a noninvasive system that identifies liver fibrosis in patients with NAFLD. Hepatology. 2007; 45: 846-854.

27. Ishak K, Baptista A, Bianchi L, et al. Histological grading and staging of chronic hepatitis. Journal of hepatology. 1995; 22: 696-699.

28. Elsheikh M, Wass JA \& Conway GS. Autoimmune thyroid syndrome in women with Turner's syndrome the association with karyotype. Clin Endocrinol (Oxf). 2001; 55: 223-226.

29. El-Mansoury M, Bryman I, Berntorp K, Hanson C, Wilhelmsen L \& Landin-Wilhelmsen K. Hypothyroidism is common in Turner syndrome: results of a five-year follow-up. J Clin Endocrinol Metab. 2005; 90: 21312135 .

30. Roulot D, Malan V, Ziol M, et al. High frequency of X chromosome abnormalities in women with short stature and elevated liver enzymes. J Clin Endocrinol Metab. 2014; 99: 1592-1596.

31. Caprio M, Infante M, Calanchini M, Mammi C \& Fabbri A. Vitamin D: not just the bone. Evidence for beneficial pleiotropic extraskeletal effects. Eat Weight Disord. 2017; 22: 27-41.

32. Targher G, Bertolini L, Scala L, et al. Associations between serum 25-hydroxyvitamin D3 concentrations and liver histology in patients with non-alcoholic fatty liver disease. Nutr Metab Cardiovasc Dis. 2007; 17: $517-524$.

33. Gaertner S, Jeandidier N, Glasser L, Ohl J, Trinh A \& Stephan D. Turner's syndrome: is there a risk of widespread vascular abnormalities? Clin Endocrinol (Oxf). 2016; 84 634-635.

34. Wanless IR. Micronodular transformation (nodular regenerative hyperplasia) of the liver: a report of 64 cases among 2,500 autopsies and a new classification of benign hepatocellular nodules. Hepatology. 1990; 11: 787-797.

35. Messina MF, Squadrito G, Valenzise M, et al. Fibroscan: a new noninvasive method for evaluation of liver dysfunction in Turner syndrome. Eur J Clin Invest. 2011; 41: 183-188. 
Table 1 Main clinical, genetic and biochemical characteristics in TS population

\begin{tabular}{|c|c|c|}
\hline & $\begin{array}{c}\text { TS patients } \\
\quad N=125\end{array}$ & \\
\hline Age, years & 35 & $18-73$ \\
\hline Age of TS diagnosis, years & 12 & $0-62$ \\
\hline $\begin{array}{l}\text { Karyotype } \\
\text { - 45,X } \\
\text { - 45,X/46,XX, 45,X/47,XXX, 45,X/46,XX/47,XXX } \\
\text { - Del(Xp) } \\
\text { - Del(Xq) } \\
\text { - Iso(Xq) } \\
\text { - RingX } \\
\text { - Y } \\
\text { - Other }\end{array}$ & $\begin{array}{c}51 \\
18 \\
4 \\
2 \\
12 \\
11 \\
7 \\
13\end{array}$ & $\begin{array}{c}43.2 \% \\
15.2 \% \\
3.4 \% \\
1.7 \% \\
10.2 \% \\
9.3 \% \\
5.9 \% \\
11 \%\end{array}$ \\
\hline Height, cm & 149.8 & $115-172$ \\
\hline Weight, kg & 59 & $24.4-105.4$ \\
\hline $\mathrm{BMI}, \mathrm{kg} / \mathrm{m}^{2}$ & 25.8 & $14.6-51.3$ \\
\hline Previous GH & 60 & $48 \%$ \\
\hline HRT duration, years ${ }^{\#}$ & 12 & $0-43$ \\
\hline Type 2 diabetes & 13 & $10.4 \%$ \\
\hline Hypertension & 34 & $27.2 \%$ \\
\hline Dyslipidaemia & 19 & $15.2 \%$ \\
\hline Autoimmune disease & $\begin{array}{c}38 \\
30 \mathrm{TH} \\
10 \text { Celiac } \\
9 \text { Others }\end{array}$ & $30.4 \%$ \\
\hline Bicuspid aortic valve (BAV) & 24 & $19.2 \%$ \\
\hline Coarctation aorta (CA) & 11 & $8.8 \%$ \\
\hline AA diameter, cm & 2.8 & $2-4.4$ \\
\hline AA ASI, $\mathrm{cm} / \mathrm{m}^{2}$ & 1.7 & $1.09-3.16$ \\
\hline Aortic sinuses diameter, cm & 2.9 & $1.9-4.1$ \\
\hline Aortic sinuses ASI, cm/m² & 1.85 & $1.24-3.1$ \\
\hline
\end{tabular}

Notes: Continuous variables are expressed as median (range) and categorical variables are expressed as number of patients (percentage). Abbreviations: BMI, body mass index; $\mathrm{GH}$, growth hormone treatment; HRT, hormone replacement therapy; AA, ascending aorta; ASI, aortic size index; TH, Hashimoto's thyroiditis. \# A total of 16 women had spontaneous menarche and were not treated with HRT. 
Table 2 Clinical and biochemical differences between TS patients with normal and elevated GGT levels

\begin{tabular}{|c|c|c|c|}
\hline & $\begin{array}{c}\text { Normal GGT } \\
\qquad \begin{array}{c}N=58\end{array}\end{array}$ & $\begin{array}{l}\text { Elevated GGT } \\
\qquad \mathrm{N}=56\end{array}$ & \\
\hline Age, years & $33.1 \pm 11.2$ & $40.4 \pm 14.6$ & $0.004^{* *}$ \\
\hline Age at diagnosis, years & $14.6 \pm 9.8$ & $13.6 \pm 14.3$ & 0.962 \\
\hline Height, cm & $151.7 \pm 7.3$ & $147.8 \pm 6.8$ & $0.004^{* *}$ \\
\hline Weight, kg & $61.2 \pm 13.5$ & $63.2 \pm 15.2$ & 0.483 \\
\hline BMI, $\mathrm{kg} / \mathrm{m}^{2}$ & $26.6 \pm 6$ & $28.7 \pm 6.7$ & 0.084 \\
\hline GH therapy, \% & 49.1 & 47.3 & 0.845 \\
\hline HRT duration, years & $11.7 \pm 9.9$ & $18.8 \pm 12.2$ & $0.001^{* *}$ \\
\hline Type 2 diabetes, \% & 6.9 & 16.1 & 0.123 \\
\hline $\mathrm{HbA1c}, \mathrm{mmol} / \mathrm{mol}$ & $33.8 \pm 6.7$ & $34.8 \pm 12.2$ & 0.569 \\
\hline Hypertension, \% & 25.9 & 33.9 & 0.347 \\
\hline Dyslipidaemia, \% & 12.1 & 21.4 & 0.180 \\
\hline Tot-C, $\mathrm{mmol} / \mathrm{L}$ & $5 \pm 1.1$ & $5.4 \pm 1.1$ & 0.069 \\
\hline LDL-C, mmol/L & $2.8 \pm 0.8$ & $3 \pm 0.9$ & 0.41 \\
\hline HDL-C, mmol/L & $1.7 \pm 0.5$ & $1.8 \pm 0.5$ & 0.481 \\
\hline TG, mmol/L & $1.1 \pm 0.6$ & $1.4 \pm 0.8$ & $0.044^{*}$ \\
\hline TPOAb, \% & 25.9 & 25 & 0.916 \\
\hline Thyroxine, \% & 24.1 & 25 & 0.915 \\
\hline Celiac disease, $\%$ & 10.3 & 5.4 & 0.490 \\
\hline BAV, \% & 10.5 & 23.6 & 0.065 \\
\hline CA, \% & 6.9 & 10.9 & 0.521 \\
\hline Aortic sinuses diameter, cm & $2.8 \pm 0.34$ & $2.95 \pm 0.38$ & $0.037^{*}$ \\
\hline Aortic sinuses ASI, cm/m² & $1.78 \pm 0.26$ & $1.9 \pm 0.35$ & 0.059 \\
\hline AA diameter, cm & $2.68 \pm 0.38$ & $2.94 \pm 0.55$ & $0.007^{\star \star}$ \\
\hline AA ASI, $\mathrm{cm} / \mathrm{m}^{2}$ & $1.72 \pm 0.3$ & $1.9 \pm 0.41$ & $0.011^{*}$ \\
\hline
\end{tabular}

Notes: Continuous variables are expressed as mean (SD) and categorical variables are expressed as percentage. Abbreviations: BMI, body mass index; GH, growth hormone treatment; HRT, hormone replacement therapy; Tot-C, total cholesterol; LDL-C, low-density lipoprotein cholesterol; HDL-C, high-density lipoprotein cholesterol; TG, tryglycerides; TPOAb, thyroid peroxidase antibodies; BAV, bicuspid aortic valve; CA, coarctation of the aorta; AA, ascending aorta; ASI, aortic size index. ${ }^{*} P<0.05,{ }^{* *} P<0.01$. 
Table 3 Clinical and biochemical differences between TS patients with normal and elevated aminotransferases and FIB-4 index

\begin{tabular}{|c|c|c|c|}
\hline & $\begin{array}{c}\text { Normal } \\
\text { ALT/AST/FIB4 } \\
\mathrm{N}=94\end{array}$ & $\begin{array}{c}\text { Elevated } \\
\text { ALT/AST/FIB4 } \\
\mathrm{N}=31\end{array}$ & \\
\hline Age, years & $34.3 \pm 13.6$ & $39.1 \pm 13.2$ & 0.086 \\
\hline Age at diagnosis, years & $13 \pm 10.4$ & $14.9 \pm 15.6$ & 0.544 \\
\hline Height, cm & $150.2 \pm 8.4$ & $148.4 \pm 6.5$ & 0.273 \\
\hline Weight, kg & $62 \pm 13.8$ & $59.8 \pm 17.3$ & 0.472 \\
\hline BMI, $\mathrm{kg} / \mathrm{m}^{2}$ & $27.4 \pm 5.9$ & $27.1 \pm 7.9$ & 0.862 \\
\hline GH therapy, \% & 47.8 & 56.7 & 0.399 \\
\hline HRT duration, years & $14.5 \pm 11.5$ & $14.8 \pm 12.1$ & 0.892 \\
\hline Type 2 diabetes, $\%$ & 10.9 & 9.7 & 0.852 \\
\hline $\mathrm{HbA1c}, \mathrm{mmol} / \mathrm{mol}$ & $33.8 \pm 6.7$ & $34.8 \pm 12.2$ & 0.569 \\
\hline Hypertension, \% & 27.5 & 29 & 0.867 \\
\hline Dyslipidaemia, \% & 16.3 & 12.9 & 0.779 \\
\hline Tot-C, mmol/L & $5 \pm 1.1$ & $5.4 \pm 1.2$ & 0.097 \\
\hline LDL-C, mmol/L & $2.8 \pm 0.8$ & $3 \pm 0.9$ & 0.313 \\
\hline HDL-C, mmol/L & $1.7 \pm 0.5$ & $1.9 \pm 0.5$ & $0.043^{*}$ \\
\hline TG, mmol/L & $1.2 \pm 0.7$ & $1.3 \pm 0.7$ & 0.718 \\
\hline TPOAb, \% & 23.1 & 29 & 0.506 \\
\hline Thyroxine, \% & 22 & 32.3 & 0.251 \\
\hline Celiac disease, $\%$ & 11 & 0 & 0.063 \\
\hline BAV, \% & 19.6 & 20 & 0.959 \\
\hline CA, \% & 8.9 & 9.7 & 0.895 \\
\hline Aortic sinuses diameter, cm & $2.87 \pm 0.34$ & $3.06 \pm 0.36$ & $0.043^{*}$ \\
\hline Aortic sinuses ASI, $\mathrm{cm} / \mathrm{m}^{2}$ & $1.82 \pm 0.32$ & $2.02 \pm 0.35$ & $0.025^{\star}$ \\
\hline AA diameter, $\mathrm{cm}$ & $2.83 \pm 0.48$ & $3.02 \pm 0.4$ & 0.078 \\
\hline AA ASI, $\mathrm{cm} / \mathrm{m}^{2}$ & $1.83 \pm 0.39$ & $2.02 \pm 0.32$ & $0.032^{*}$ \\
\hline
\end{tabular}

Notes: Continuous variables are expressed as mean (SD) and categorical variables are expressed as percentage. Abbreviations: BMI, body mass index; $\mathrm{GH}$, growth hormone treatment; HRT, hormone replacement therapy; Tot-C, total cholesterol; LDL-C, low-density lipoprotein cholesterol; HDL-C, high-density lipoprotein cholesterol; TG, tryglycerides; TPOAb, thyroid peroxidase antibodies; BAV, bicuspid aortic valve; CA, coarctation of the aorta; AA, ascending aorta; ASI, aortic size index. ${ }^{*} \mathrm{P}<0.05$ 
(a)

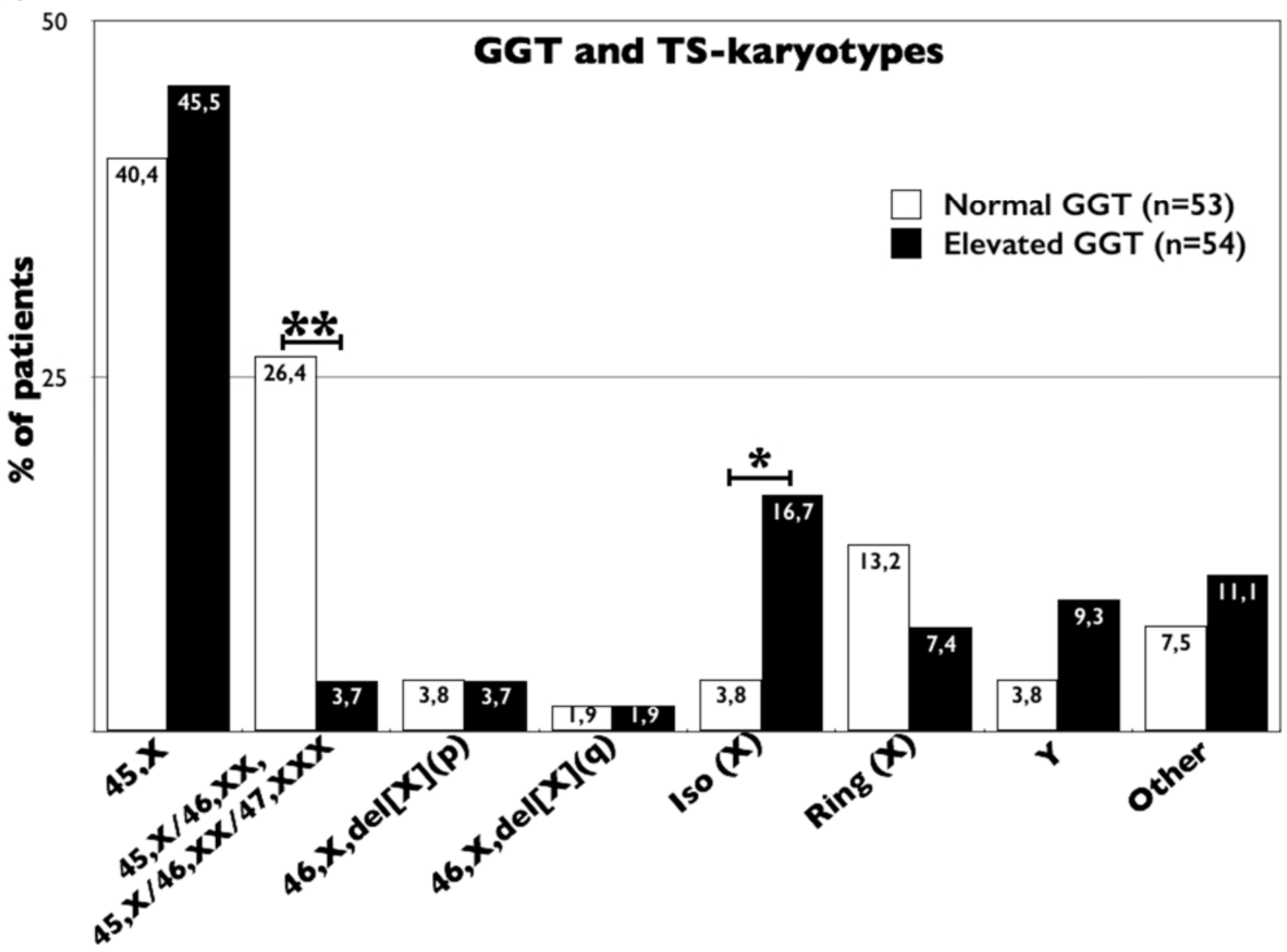

Abbreviations: GGT, gamma-glutamyl transferase. ${ }^{*} P<0.05,{ }^{* *} P<0.01$. 


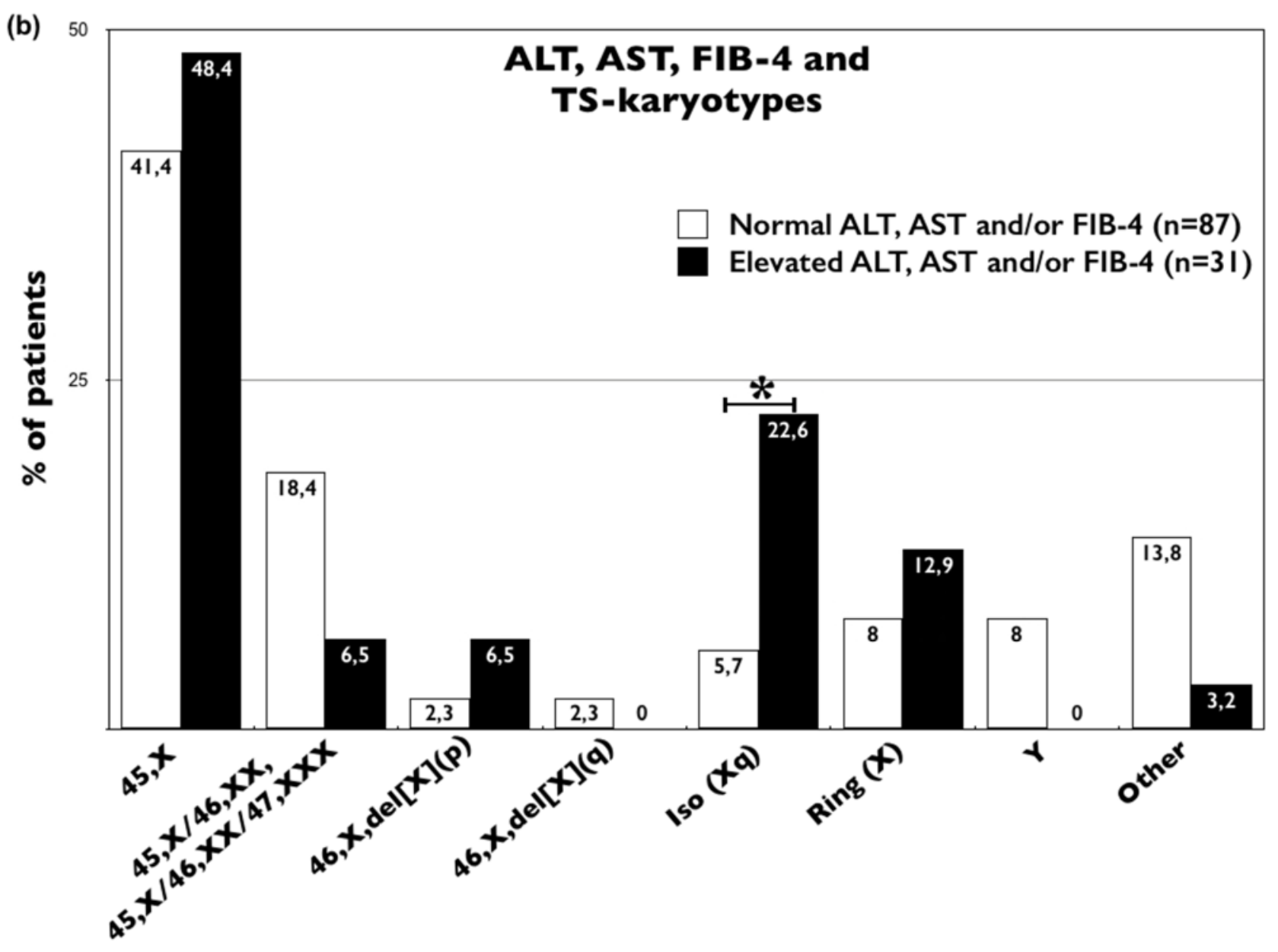

Abbreviations: ALT, alanine aminotransferase; AST, aspartate aminotransferase; FIB-4, Fibrosis-4 score. ${ }^{*} P<0.05$. 